\title{
Extended Use of Hypothermia in Elderly Patients with Malignant Cerebral Edema as an Alternative to Hemicraniectomy
}

\author{
Han-Yeong Jeong, ${ }^{\mathrm{a}^{*}}$ Jun-Young Chang, ${ }^{\mathrm{b}^{*}}$ Kyu Sun Yum, ${ }^{\mathrm{a}}$ Jeong-Ho Hong, ${ }^{\mathrm{c}}$ Jin-Heon Jeong, ${ }^{\mathrm{d}}$ Min-Ju Yeo, \\ Hee-Joon Bae, ${ }^{\text {a }}$ Moon-Ku Han, ${ }^{a}$ Kiwon Lee ${ }^{\mathrm{f}}$ \\ a'Department of Neurology, Seoul National University Bundang Hospital, Seongnam, Korea \\ bDepartment of Neurology, Gyeongsang National University Changwon Hospital, Changwon, Korea \\ 'Department of Neurology, Keimyung University Dongsan Medical Center, Daegu, Korea \\ ${ }^{\mathrm{d}}$ Department of Intensive Care Medicine and Neurology, Dong-A University Hospital, Busan, Korea \\ 'Department of Neurology, Chungbuk National University, Chungju, Korea \\ fDepartment of Neurology and Neurosurgery, University of Texas Houston Medical School and Memorial Hermann Texas Medical Center, \\ Houston, United States
}

Background and Purpose The use of decompressive hemicraniectomy (DHC) for the treatment of malignant cerebral edema can decrease mortality rates. However, this benefit is not sufficient to justify its use in elderly patients. We investigated the effects of therapeutic hypothermia (TH) on safety, feasibility, and functional outcomes in elderly patients with malignant middle cerebral artery (MCA) infarcts.

Methods Elderly patients 60 years of age and older with infarcts affecting more than two-thirds of the MCA territory were included. Patients who could not receive DHC were treated with TH. Hypothermia was started within 72 hours of symptom onset and was maintained for a minimum of 72 hours with a target temperature of $33^{\circ} \mathrm{C}$. Modified Rankin Scale (mRS) scores at 3 months following treatment and complications of $\mathrm{TH}$ were used as functional outcomes.

Results Eleven patients with a median age of 76 years and a median National Institutes of Health Stroke Scale score of 18 were treated with $\mathrm{TH}$. The median time from symptom onset to initiation of TH was $30.3 \pm 23.0$ hours and TH was maintained for a median of $76.7 \pm 57.1$ hours. Shivering $(100 \%)$ and electrolyte imbalance (82\%) were frequent complications. Two patients died (18\%). The mean mRS score 3 months following treatment was $4.9 \pm 0.8$.

Conclusions Our results suggest that extended use of hypothermia is safe and feasible for elderly patients with large hemispheric infarctions. Hypothermia may be considered as a therapeutic alternative to DHC in elderly individuals. Further studies are required to validate our findings.
Correspondence: Moon-Ku Han Department of Neurology, Seoul National University Bundang Hospital, 82 Gumi-ro 173beon-gil, Bundang-gu, Seongnam 13620, Korea

Tel: +82-31-787-7464

Fax: +82-31-787-4059

Email:mkhan@snu.ac.kr

Received: April 12, 2016

Revised: May 3, 2016

Accepted: May 4, 2016

*Han-Yeong Jeong and Jun-Young Chang contributed equally to this research as first author.

The authors have no financial conflicts of interest.

Keywords Cerebral infarction; Hypothermia; Hemicraniectomy; Elderly; Brain edema

\section{Introduction}

Large hemispheric infarction is a devastating type of stroke accounting for up to $10 \%$ of all supratentorial ischemic strokes and has mortality rates of up to $80 \% 0^{1,2}$ The severe mass effect produced by progressive swelling of the infarcted brain tissue and the subsequent inevitable elevation of intracranial pressure typically result in poor neurological outcomes. ${ }^{3}$ Conventional medical management, including osmotic therapy, hyperventilation, and barbiturate coma, has not been shown to improve 
long-term functional outcomes. ${ }^{4-6} \mathrm{~A}$ pooled analysis of three randomized, controlled trials has shown that early decompressive hemicraniectomy (DHC) is beneficial for the treatment of malignant cerebral edema. ${ }^{7-10}$ However, approximately $40 \%$ of patients with large hemispheric infarctions are older than 60 years $^{11,12}$ and the upper age limit in the above analysis was 60.

Recently, a randomized trial demonstrated that $\mathrm{DHC}$ improved outcomes and reduced mortality rates in patients older than 60 with large middle cerebral artery (MCA) infarctions. ${ }^{13}$ The 6-month mortality rate was lower for patients who underwent hemicraniectomy (33\%) than for those who underwent the best possible medical therapy with standardized intensive care (70\%). However, DHC was less effective in reducing the mortality rate in older patients than in younger ones (33\% vs. 50\%).

Older patients with multiple underlying comorbidities may not tolerate general anesthesia or surgical treatment, which presents a major challenge for clinicians in their decision-making process. Moreover, due to cultural difference in attitudes toward surgery in Korea, some patients or caregivers often refuse to receive major surgery, even if it is not contraindicated in their case.

The Cochrane Collaboration Review analyzed eight trials of cooling therapy for the treatment of acute ischemic stroke and reported that therapeutic hypothermia (TH) had no clear benefit in reducing the rates of mortality or functional dependency and was associated with increased risks for cardiac arrhythmias and infections, which have limited its use in clinical practice. ${ }^{14-16}$ However, the hypothermic state may activate a number of potentially neuroprotective mechanisms, possibly reducing neurological injury and decreasing cytotoxic edema by preserving cerebrovascular autoregulation. ${ }^{17-19}$ The concept of TH has also been suggested to be a promising approach in reducing global cerebral edema and intracranial hypertension due to acute ischemic and hemorrhagic brain injury. ${ }^{5,20}$

Hypothermia treatment may be an alternative therapeutic option for patients with large cerebral infarctions who cannot receive DHC. Our study aimed to investigate whether TH can safely reduce or halt the progression of malignant cerebral edema in an elderly population with large hemispheric infarctions.

\section{Methods}

\section{Patients}

DHC was the first-line therapy for patients with large hemispheric infarcts. However, those patients who declined DHC were then counseled about the use of $\mathrm{TH}$ as an alternative treatment option. Written informed consent was obtained from all caregivers prior to patient enrollment into TH treatment. This investigation was approved by the institutional review board.

\section{Patient population}

We retrospectively reviewed the clinical and radiographic data from elderly patients aged 60 years or older who were diagnosed with a large acute hemispheric infarction and were treated with TH between February 2011 and August 2012. Seoul National University Bundang Hospital's stroke data registry was used for analysis. Patients were enrolled if they met all of the following criteria: age $\geq 60$ years, with acute ischemic stroke, hospital admission within 12 hours of stroke symptom onset, and initial National Institutes of Health Stroke Scale (NIHSS) score $>10$. Specific neuroimaging criteria included the following: large hemispheric infarction involving more than two-thirds of the MCA territory, involvement of the basal ganglia on diffusionweighted magnetic resonance imaging scans within 12 hours of symptom onset, and parenchymal hypodensity involving more than two-thirds of the MCA territory with a midline shift evident on computed tomography (CT) scans within 72 hours of onset.

Patients were ineligible if they demonstrated any of the following exclusion criteria: severe cardiac dysarrhythmia or a corrected OT (OTc) interval $>450 \mathrm{~ms}$, platelet count $<75,000 / \mathrm{mm}^{3}$, known coagulopathy (prothrombin time international normalized ratio $>1.3$ ) or active bleeding, severe sepsis and septic shock, preexisting illness with a life expectancy of $<6$ months, previous modified Rankin Scale (mRS) score $>4$, concurrent monoamine oxidase inhibitor usage, or pregnancy. ${ }^{21}$

\section{Data collection}

We reviewed clinical data, including patient demographics, diagnosis, Trial of ORG 10172 in Acute Stroke Treatment classification, vascular risk factors (hypertension, diabetes mellitus, hyperlipidemia, smoking, previous stroke, and previous cardiac disorders including arrhythmia), stroke severity as measured by NIHSS score at baseline and discharge, and long-term functional outcome 3 months following treatment as measured by the mRS.

Radiologic data included an initial magnetic resonance imaging scan on admission and follow-up computed tomography (CT) scans before and after TH. The follow-up CT scans were used to detect any hemorrhagic transformation and to quantify the magnitude of the midline shift 1 day and 3 days after completing TH. Hemorrhagic transformation was categorized into four different subtypes (hemorrhagic infarction 1 or 2, and parenchymal hemorrhage 1 or 2) according to the European Cooperative Acute Stroke Study I and II criteria. ${ }^{22,23}$ The progression of cerebral edema was quantified by measuring lateral displacement of the calcified pineal gland from the midline on CT scans. ${ }^{24}$

Physiological data included heart rate, blood pressure, and body temperature, which were measured every hour. Timeline data consisted of admission and discharge date, stroke onset, 
time of symptom onset to $\mathrm{TH}$ initiation, and time to target temperature. Laboratory data included white blood cell count, hematocrit and hemoglobin levels, platelet count, electrolytes (sodium, potassium, chloride, and phosphate), glucose, creatinine, albumin, creatinine kinase, aspartate transaminase, alanine transaminase, prothrombin time, activated partial thromboplastin time, and arterial blood gas. Urinalysis, electrocardiography, and chest radiography were also performed. Daily chest radiographs and microbiological pancultures (urine, blood, and sputum) were reviewed for infection status. Adverse events were adapted from the National Acute Brain Injury Study, ${ }_{1}^{25}$ which included cardiovascular complications, infections, electrolyte imbalances, and bleeding complications or coagulopathies, pulmonary edema, and liver function abnormalities.

\section{Clinical assessment}

Neurological deficits on admission and discharge were assessed using the NIHSS score. The functional status of each patient was assessed 3 months after treatment using mRS scoring. All clinical assessments were recorded by cerebrovascular neurologists. Complications arising from TH were recorded to determine safety and feasibility outcomes.

\section{Hypothermia treatment}

All patients were admitted to the neurological intensive care unit and treated according to the standardized institutional hypothermia protocol after obtaining informed consent. Patients were cooled with an external cooling device using cold watercirculating wrapping garments (Arctic Sun Temperature Management System; Medivance, Louisville, CO, USA) and subsequent use of $500 \mathrm{~mL}$ to $1 \mathrm{~L}$ of cold $\left(4.0^{\circ} \mathrm{C}\right)$ saline infused intravenously within 30 to 60 minutes after enrollment. Core temperature was measured with an esophageal probe. After the core temperature reached the target temperature of $33^{\circ} \mathrm{C}$, the Arctic Sun system automatically maintained the target temperature by regulating the temperature of the circulating water. Core temperature was continuously monitored and was recorded every hour. Invasive monitoring equipment, such as an arterial line or a central venous line, was used for those who needed close hemodynamic monitoring.

All patients treated with TH were intubated and adequately sedated. We adopted the Columbia University Anti-shivering Protocol, ${ }^{26}$ which included both universal and patient-specific therapies. Initially, acetaminophen (650 mg every 4 hours) and buspirone (30 mg every 8 hours) were used as anti-shivering agents, and a skin counter-warming system (Bear Hugger, Arizant Healthcare Inc.; Eden Prairie, MN, USA) was applied. If shivering developed, meperidine (50-100 mg bolus followed by $12.5-$
$50 \mathrm{mg} / \mathrm{h}$ ) or propofol infusions (75-300 mg/h) were used. If shivering persisted, a dexmedetomidine infusion (loading dose $1 \mu \mathrm{g}$ / $\mathrm{kg}$ over 10 minutes, followed by an infusion of $0.3-1.5 \mu \mathrm{g} / \mathrm{kg} / \mathrm{h}$ ) was used, and finally, a continuous infusion of vecuronium (0.1 $\mathrm{mg} / \mathrm{kg} / \mathrm{h}$ ) was administered for refractory shivering cases.

All patients underwent follow-up CT scans before and after TH to visually assess the degree of cerebral edema. After obtaining stable clinical and CT findings for 24 hours, a passive and slow rewarming process was initiated. The rate of rewarming was set at $0.1^{\circ} \mathrm{C} / \mathrm{h}$.

\section{Results}

\section{Patient characteristics}

A total of 199 patients with MCA infarctions between February 2011 and August 2012 were screened. Thirty-four patients had malignant MCA infarctions. Five patients (15\%) underwent DHC, 13 (38\%) received conservative treatment, 16 (47\%) were treated with TH, and 11 (32\%) were eligible for our study. Baseline patient characteristics are summarized in Table 1. The median age was 76 (range, 64-82 years) and the median NIHSS score was 18 (range, 13-22) upon admission. Six (55\%) of the 11 patients underwent intravenous or intra-arterial thrombolysis. Based on Trial of ORG 10,172 in Acute Stroke Treatment subtype classification, 6 (55\%) of 11 patients had a cardioembolism, 3 (27\%) had large artery atherosclerosis, and 2 (18\%) were of undetermined etiology. One patient had two potential causes of stroke (large artery atherosclerosis and cardioembolism), and the

Table 1. Characteristics of patients who underwent therapeutic hypothermia for a large hemispheric infarction

\begin{tabular}{lc}
\hline Characteristics & $\mathrm{N}=11$ \\
\hline Age, median (range), (year) & $76(64-82)$ \\
Male, $\mathrm{n}(\%)$ & $6(55)$ \\
Initial NIHSS, median (range) & $18(13-22)$ \\
Thrombolysis (IV or IA), $\mathrm{n}(\%)$ & $6(55)$ \\
Stroke subtype, $\mathrm{n}(\%)$ & \\
Cardioembolism & $6(55)$ \\
Large artery atherosclerosis & $3(27)$ \\
Undetermined cause & $2(18)$ \\
Risk factors, $\mathrm{n}(\%)$ & \\
Hypertension & $9(82)$ \\
Diabetes mellitus & $6(55)$ \\
Hyperlipidemia & $2(18)$ \\
Atrial fibrillation & $6(55)$ \\
Coronary artery disease & $4(36)$ \\
Previous stroke & $3(27)$ \\
Smoking & $5(45)$ \\
Time to hypothermia, median (range), (hour) & $30.3(4-72)$ \\
Mortality, $\mathrm{n}(\%)$ & $2(18)$ \\
\hline
\end{tabular}

$I V$, intravenous; IA, intra-arterial. 
Table 2. Basic characteristics, vascular pathology, stroke severity, and feasibility of therapeutic hypothermia for patients with a large hemispheric infarction

\begin{tabular}{|c|c|c|c|c|c|c|c|c|c|c|c|}
\hline \multirow{2}{*}{$\begin{array}{l}\text { Patient } \\
\text { no. }\end{array}$} & \multirow{2}{*}{ Age } & \multirow{2}{*}{ Sex } & \multirow{2}{*}{$\begin{array}{l}\text { Arterial occlusion } \\
\text { site }\end{array}$} & \multirow{2}{*}{ Lesion site } & \multirow{2}{*}{ HT } & \multirow{2}{*}{$\begin{array}{l}\text { Stroke } \\
\text { subtype }\end{array}$} & \multirow{2}{*}{$\begin{array}{l}\text { Initial } \\
\text { NIHSS }\end{array}$} & \multirow{2}{*}{$\begin{array}{l}\text { Prestroke } \\
\text { mRS }\end{array}$} & \multirow{2}{*}{ Thrombolysis } & \multicolumn{2}{|c|}{ Hypothermia (hour) } \\
\hline & & & & & & & & & & Onset to $T x$ & Duration \\
\hline 1 & 69 & $\mathrm{~F}$ & Lt dICA & MCA & - & CE & 16 & 0 & $\mathrm{IV} / \mathrm{IA}$ & 72 & 72 \\
\hline 2 & 64 & $\mathrm{~F}$ & Lt pICA & MCA & - & UD & 22 & 0 & - & 71 & 46 \\
\hline 3 & 80 & $\mathrm{~F}$ & Rt pMCA & MCA & $\mathrm{HI}-2$ & UD & 17 & 3 & - & 36 & 75 \\
\hline 4 & 82 & M & Rt pMCA & MCA & $\mathrm{PH}-2$ & CE & 21 & 0 & - & 36 & 77 \\
\hline 5 & 76 & M & Rt pMCA & MCA & - & CE & 18 & 0 & $\mathrm{IV} / \mathrm{IA}$ & 30 & 72 \\
\hline 6 & 74 & M & Lt pMCA & MCA & $\mathrm{PH}-2$ & CE & 21 & 2 & - & 31 & 73 \\
\hline 7 & 66 & M & Lt dICA & MCA & - & LAA & 20 & 0 & $\mathrm{IV} / \mathrm{IA}$ & 14 & 143 \\
\hline 8 & 79 & $\mathrm{~F}$ & Rt plCA & $\mathrm{MCA}+\mathrm{ACA}$ & $\mathrm{HI}-2$ & $\mathrm{CE}$ & 16 & 0 & IV & 4 & 125 \\
\hline 9 & 66 & M & Lt plCA & MCA & - & LAA & 22 & 0 & IV & 14 & 254 \\
\hline 10 & 80 & $F$ & Rt dICA & MCA & $\mathrm{HI}-2$ & LAA & 13 & 0 & $\mathrm{IV} / \mathrm{IA}$ & 16 & 107 \\
\hline 11 & 80 & M & Rt pMCA & MCA & - & CE & 17 & 0 & - & 12 & 104 \\
\hline $\begin{array}{l}\text { Median } \\
\text { (SD) }\end{array}$ & $\begin{array}{l}76.0 \\
(6.7)\end{array}$ & & & & & & $\begin{array}{l}18.0 \\
(2.9)\end{array}$ & & & $\begin{array}{c}30.3 \\
(23.0)\end{array}$ & $\begin{array}{c}76.7 \\
(57.1)\end{array}$ \\
\hline
\end{tabular}

$H T$, hemorrhagic transformation; NIHSS, National Institute of Health Stroke Scale; mRS, modified Rankin Scale; onset to Tx, time of onset to initiation of hypothermia; Lt, left; Rt, right; dICA, distal internal carotid artery; pICA, proximal internal carotid artery; PMCA, proximal middle cerebral artery; ACA, anterior cerebral artery; $\mathrm{HI}$, hemorrhagic infarction; $\mathrm{PH}$, parenchymal hemorrhage; $\mathrm{CE}$, cardioembolism; UD, undetermined etiology; LAA, large artery atherosclerosis; IV, intravenous thrombolysis; IA, intra-arterial thrombolysis.

Table 3. Efficacy of therapeutic hypothermia for large hemispheric infarction

\begin{tabular}{cccccccc}
\hline \multirow{2}{*}{$\begin{array}{c}\text { Patient } \\
\text { no. }\end{array}$} & \multicolumn{2}{c}{ mRS } & & \multicolumn{3}{c}{ Pineal gland displacement $(\mathrm{mm})$} \\
\cline { 2 - 3 } \cline { 6 - 7 } & Prestroke & 3 months & & First day & Post-hypothermia & Change \\
\hline 1 & 0 & 5 & & 5.5 & 6.0 & 0.5 \\
2 & 0 & 6 & & 12.0 & - & - \\
3 & 3 & 4 & & 2.5 & 2.5 & 0 \\
4 & 0 & 5 & & 9.5 & 8.5 & -1.0 \\
5 & 0 & 3 & & 3.5 & 3.5 & 0 \\
6 & 2 & 5 & & 2.5 & 2.5 & 0 \\
7 & 0 & 5 & & 6.5 & 5.0 & -1.5 \\
8 & 0 & 5 & & 3.5 & 11.0 & 7.5 \\
9 & 0 & 5 & & 6.9 & 12.5 & 5.6 \\
10 & 0 & 6 & & 0.0 & - & - \\
11 & 0 & 5 & 0.0 & 0.0 & 0 \\
\hline
\end{tabular}

mRS, modified Rankin Scale.

other patient was not fully evaluated due to death (undetermined etiology, negative evaluation). Nine (82\%) of 11 patients exhibited hypertension and $3(27 \%)$ had previously experienced stroke. Premorbid mRS scores were 0 for 9 patients, 2 for 1 patient, and 3 for 1 patient. Hemorrhagic transformation was detected in $5(45 \%)$ of 11 patients before the initiation of hypothermia, and $4(80 \%)$ out of these 5 patients experienced an additional mass effect due to hemorrhagic transformation (parenchymal hemorrhage 1 or 2 ).

\section{Feasibility of therapeutic hypothermia}

TH was initiated at a median of $30.3 \pm 23.0$ hours after stroke onset (Table 2). The median time to reach core target temperature $\left(33^{\circ} \mathrm{C}\right)$ after cold saline infusion was $4.0 \pm 2.4$ hours. TH was
Table 4. Complications of therapeutic hypothermia

\begin{tabular}{ll}
\hline Characteristics & \\
\hline Shivering, $\mathrm{n}(\%)$ & $11(100)$ \\
Cardiovascular complications, $\mathrm{n}(\%)$ & \\
Bradycardia & $5(45)$ \\
Arrhythmia & $5(45)$ \\
Ventricular tachycardia & $2(18)$ \\
Rapid ventricular response & $3(27)$ \\
Hypotension & $2(18)$ \\
Infection, $\mathrm{n}(\%)$ & \\
Pneumonia & $6(55)$ \\
Acute cholecystitis & $1(9)$ \\
Sepsis & $0(0)$ \\
Electrolyte imbalance, $\mathrm{n}(\%)$ & \\
Hypernatremia $(>145 \mathrm{mmol} / \mathrm{L})$ & $5(45)$ \\
Hyponatremia $(<135 \mathrm{mmol} / \mathrm{L})$ & $1(9)$ \\
Hypokalemia $(<3.5 \mathrm{mmol} / \mathrm{L})$ & $9(82)$ \\
Hypophosphatemia $(<2.5 \mathrm{mmol} / \mathrm{L})$ & $9(82)$ \\
Bleeding complications, $\mathrm{n}(\%)$ & \\
Hemoptysis & $2(18)$ \\
Upper gastrointestinal bleeding & $1(9)$ \\
Hematuria & $2(18)$ \\
Pulmonary edema & $3(27)$ \\
\hline
\end{tabular}

maintained for a median of $76.7 \pm 57.1$ hours (range, $46-254$ hours).

\section{Outcomes}

The mean mRS score 3 months after treatment was $4.9 \pm 0.8$ for all patients and $4.7 \pm 0.7$ for survivors (Table 3 ). There were two deaths (18\%) due to either progression of cerebral edema (case 2) or sudden cardiac arrhythmia after rewarming (case 10). 
One (9\%) of the 11 patients treated with TH achieved a favorable functional outcome (mRS 3).

All patients who exhibited pineal gland displacement $>5 \mathrm{~mm}$ on the first day of TH experienced poor outcomes (mRS 5 or 6) and the degree of the lateral displacement of the pineal gland was more significant in those who died (case 2: 12-mm pineal gland displacement). Two (33\%) of 6 patients with $<5 \mathrm{~mm}$ of pineal gland displacement exhibited an mRS of 3 or 4 three months after treatment, suggesting that the magnitude of pineal gland displacement on the first day of TH corresponded with mortality and functional outcome.

\section{Safety and complications}

Shivering was observed in all TH patients (Table 4). Bradycardia was frequently noted during the cooling phase $(45 \%, n=5)$, but was transient in nature and asymptomatic in all cases. Five patients (45\%) who had preexisting atrial fibrillation developed further cardiac arrhythmias during hypothermia, 2 (18\%) developed a non-sustained ventricular tachycardia, and 3 (27\%) experienced atrial fibrillation with rapid ventricular responses. No patient required urgent anti-arrhythmic therapy. Hypotension was noted in $2(18 \%)$ cases and was thought to be due to hypothermia-induced diuresis and relative intravascular volume depletion. Six (55\%) patients developed pneumonia, but responded favorably to appropriate antibiotic therapies.

Electrolyte imbalances were detected in most patients during TH. Serum sodium was altered in 55\% of patients (hypernatremia, $n=5$; hyponatremia, $n=1$ ). Hypokalemia was observed in $82 \%$ of patients $(n=9)$ and 1 patient developed rebound hyperkalemia during the rewarming phase. Hypophosphatemia was also a common side effect $(82 \%, n=9)$. Minor bleeding complications, such as hemoptysis $(n=2)$ and hematuria $(n=2)$, were observed, but were easily controlled. No major coagulopathy was induced by $\mathrm{TH}$.

\section{Discussion}

Our results suggest that $\mathrm{TH}$ is well-tolerated and feasible for clinical use for extended periods (i.e., $>72$ hours) in patients older than 60 years of age with large acute hemispheric infarctions.

In a pooled analysis of three European trials of hemicraniectomy (DESTINY, DECIMAL, and HAMLET) involving younger patients (age $<60$ years), mortality rates for conservative treatment and surgery were $77 \%$ and $22 \%$, respectively, at 12 months after treatment. ${ }^{7}$ In patients older than 60 years of age (median, 70 years; range, 61-82 years), hemicraniectomy reduced 6-month mortality by $33 \%$ and 1 -year mortality by $43 \%$, as compared to $70 \%$ and $76 \%$ in a control group. ${ }^{13}$ Our overall $18 \%$ mortality rate in elderly patients treated with $\mathrm{TH}$ is comparable to the results of a previous pooled analysis of DHC (22\%) in young patients, but slightly higher than that of a study by Georgiadis et al., ${ }^{27}$ who reported a $12 \%$ mortality rate in 17 patients who received DHC. The above randomized controlled trials were conducted with relatively young patients ( $<60$ years old in most cases) and the mortality rate of patients who underwent DHC was notably high at approximately $20-25 \% .^{8-10}$ Although our study is not appropriately powered, our results indicate that $\mathrm{TH}$ may be a safe and feasible alternative to DHC in elderly patients with malignant hemispheric edema, especially given the inevitable risks and potential complications associated with surgery.

The feasibility and safety of hypothermia were previously demonstrated in the literature. ${ }^{15,16}$ The ICTuS trial showed that hypothermia using an endovascular method decreased acute post-ischemic cerebral edema. ${ }^{20}$ The authors of that trial demonstrated the feasibility and safety of combining endovascular hypothermia with intravenous thrombolysis. ${ }^{28}$ Although the duration of hypothermia was limited to 72 hours in the previous studies, we opted to continue cooling for much longer durations (up to 254 hours) in a larger number of patients. One patient (case 9) initially underwent TH for 155 hours with clinical success in halting malignant edema. Rebound intracranial pressure crises and edema were not infrequent and were observed during the passive rewarming phase. As a result, TH was restarted for an additional 99 hours for the patient who developed a recurrence of cerebral edema, as indicated by clinical and radiographic data.

As observed in previous studies, our results demonstrate that $\mathrm{TH}$ is associated with a number of specific complications, such as pneumonia, bradycardia, arrhythmia, and hypokalemia, which require intensive monitoring and management in the neurological intensive care unit. In our study, the rate of complications due to $\mathrm{TH}$ increased as the duration of hypothermia increased, although all complications were well-tolerated. Six of 11 patients developed pneumonia and an increased rate of pneumonia was observed as the duration of hypothermia increased.

Our study provides a number of useful findings. First, a longer duration of TH (more than 72 hours) appeared to be feasible in these patients. Moderate hypothermia was induced effectively and maintained for more than 72 hours in all patients. Second, the timing of TH may be important. It is likely that the patient in case 2 died due to a delayed implementation of therapy. We also observed a relationship between the magnitude of pineal gland displacement (on the first day of stroke) and functional outcome: no patients with a pineal gland displacement $>10 \mathrm{~mm}$ experienced a positive outcome. However, it remains unknown whether a positive outcome could have been observed if $\mathrm{TH}$ was initiated prior to the onset of significant edema and/or severe pineal 
gland displacement.

Our study has a number of limitations. The predominant limitations are that this is a single-center, retrospective, observational study with a small sample size. A larger, multicenter, prospective study with more extensive data that exclude all potential confounders is warranted to further investigate the utility of $\mathrm{TH}$ in elderly individuals.

\section{Conclusions}

Our study provides data that are useful for the clinical decision-making process. When considering surgery for an elderly patient with a malignant MCA infarction, hypothermia may potentially be a reasonable alternative, particularly for those who may not be able to tolerate the risks of surgery. Based on our results and the available literature, a larger randomized controlled trial of hypothermia is required to validate our findings in an elderly population.

\section{References}

1. Hacke W, Schwab S, Horn M, Spranger M, De Georgia M, von Kummer R. 'Malignant' middle cerebral artery territory infarction: clinical course and prognostic signs. Arch Neurol 1996; 53:309-315.

2. Heinsius T, Bogousslavsky J, Van Melle G. Large infarcts in the middle cerebral artery territory. Etiology and outcome patterns. Neurology 1998;50:341-350.

3. Frank Jl. Large hemispheric infarction, deterioration, and intracranial pressure. Neurology 1995;45:1286-1290.

4. Hofmeijer J, van der Worp HB, Kappelle $\sqcup$. Treatment of spaceoccupying cerebral infarction. Crit Care Med 2003;31:617-625.

5. Bardutzky J, Schwab S. Antiedema therapy in ischemic stroke. Stroke 2007;38:3084-3094.

6. Juttler $E_{1}$ Schellinger PD, Aschoff A, Zweckberger $K_{1}$ Unterberg A, Hacke W. Clinical review: therapy for refractory intracranial hypertension in ischaemic stroke. Crit Care 2007;11:231.

7. Vahedi K, Hofmeijer J, Juettler E, Vicaut E, George B, Algra A, et al. Early decompressive surgery in malignant infarction of the middle cerebral artery: a pooled analysis of three randomised controlled trials. Lancet Neurol 2007;6:215-222.

8. Hofmeijer J, Kappelle $\sqcup$, Algra A, Amelink GJ, van Gijn J, van der Worp HB. Surgical decompression for space-occupying cerebral infarction (the Hemicraniectomy After Middle Cerebral Artery infarction with Life-threatening Edema Trial [HAMLET]): a multicentre, open, randomised trial. Lancet Neurol 2009;8: 326-333.

9. Vahedi K, Vicaut E, Mateo J, Kurtz A, Orabi M, Guichard JP, et al. Sequential-design, multicenter, randomized, controlled trial of early decompressive craniectomy in malignant middle cerebral artery infarction (DECIMAL Trial). Stroke 2007;38:25062517.

10. Juttler $E$, Schwab S, Schmiedek $P$, Unterberg A, Hennerici $M$, Woitzik J, et al. Decompressive Surgery for the Treatment of Malignant Infarction of the Middle Cerebral Artery (DESTINY): a randomized, controlled trial. Stroke 2007;38:2518-2525.

11. Uhl E, Kreth FW, Elias B, Goldammer A, Hempelmann RG, Liefner $M_{1}$ et al. Outcome and prognostic factors of hemicraniectomy for space occupying cerebral infarction. J Neurol Neurosurg Psychiatry 2004;75:270-274.

12. Holtkamp M, Buchheim $K$, Unterberg A, Hoffmann O, Schielke

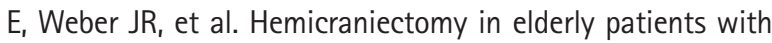
space occupying media infarction: improved survival but poor functional outcome. J Neurology Neurosurg Psychiatry 2001; 70:226-228

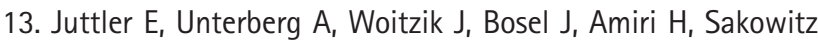
$\mathrm{OW}$, et al. Hemicraniectomy in older patients with extensive middle-cerebral-artery stroke. N Engl J Med 2014;370:10911100.

14. Den Hertog HM, van der Worp HB, Tseng MC, Dippel DW. Cooling therapy for acute stroke. Cochrane Database Syst Rev 2009:CD001247.

15. Krieger DW, De Georgia MA, Abou-Chebl A, Andrefsky JC, Sila $C A$, Katzan IL, et al. Cooling for acute ischemic brain damage (cool aid): an open pilot study of induced hypothermia in acute ischemic stroke. Stroke 2001;32:1847-1854.

16. De Georgia MA, Krieger DW, Abou-Chebl A, Devlin TG, Jauss M, Davis SM, et al. Cooling for Acute Ischemic Brain Damage (COOL AID): a feasibility trial of endovascular cooling. Neurology 2004;63:312-317.

17. Hemmen TM, Lyden PD. Multimodal neuroprotective therapy with induced hypothermia after ischemic stroke. Stroke 2009; 40(Suppl):S126-S128.

18. Polderman KH. Mechanisms of action, physiological effects, and complications of hypothermia. Crit Care Med 2009;37 (Suppl):S186-S202.

19. Linares G, Mayer SA. Hypothermia for the treatment of ischemic and hemorrhagic stroke. Crit Care Med 2009;37(Suppl): S243-S249.

20. Guluma KZ, Oh H, Yu SW, Meyer BC, Rapp K, Lyden PD. Effect of endovascular hypothermia on acute ischemic edema: morphometric analysis of the ICTuS trial. Neurocrit Care 2008;8: 42-47.

21. Dellinger RP, Levy MM, Rhodes $A$, Anane D, Gerlach H, Opal $\mathrm{SM}$, et al. Surviving Sepsis Campaigr Guidelines Committee including The Pediatric Subgroup. Surviving Sepsis Campaign: 
international guidelines for management of severe sepsis and septic shock. Intensive Care Med 2012;39:165-228.

22. Hacke $W_{1}$ Kaste $M$, Fieschi $C$, Toni $D$, Lesaffre $E_{1}$ von Kummer $R$, et al. Intravenous thrombolysis with recombinant tissue plasminogen activator for acute hemispheric stroke. The European Cooperative Acute Stroke Study (ECASS). JAMA 1995;274: 1017-1025.

23. Hacke W, Kaste $M$, Fieschi $C$, von Kummer $R$, Davalos $A$, Meier $D$, et al. Randomised double-blind placebo-controlled trial of thrombolytic therapy with intravenous alteplase in acute ischaemic stroke (ECASS II). Second European-Australasian Acute Stroke Study Investigators. Lancet 1998;352:1245-1251.

24. Ropper AH. Lateral displacement of the brain and level of consciousness in patients with an acute hemispheral mass. $N$ Engl J Med 1986;314:953-958.
25. Clifton GL, Miller ER, Choi SC, Levin HS, McCauley S, Smith KR $\mathrm{Jr}$, et al. Lack of effect of induction of hypothermia after acute brain injury. N Engl J Med 2001;344:556-563.

26. Choi HA, Ko SB, Presciutti M, Fernandez L, Carpenter AM,

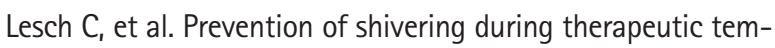
perature modulation: the Columbia anti-shivering protocol. Neurocrit Care 2011;14:389-394.

27. Georgiadis D, Schwarz S, Aschoff A, Schwab S. Hemicraniectomy and moderate hypothermia in patients with severe ischemic stroke. Stroke 2002;33:1584-1588.

28. Hemmen TM, Raman R, Guluma KZ, Meyer BC, Gomes JA, Cruz-Flores $S$, et al. Intravenous thrombolysis plus hypothermia for acute treatment of ischemic stroke (ICTUS-L): final results. Stroke 2010;41:2265-2270. 